LA W RENCE LIVERMORE NATIONAL LABORATORY

\section{(Beta)-decay experiments and the unitarity of the CKM matrix}

P. E. Garrett

December 5, 2005 
This document was prepared as an account of work sponsored by an agency of the United States Government. Neither the United States Government nor the University of California nor any of their employees, makes any warranty, express or implied, or assumes any legal liability or responsibility for the accuracy, completeness, or usefulness of any information, apparatus, product, or process disclosed, or represents that its use would not infringe privately owned rights. Reference herein to any specific commercial product, process, or service by trade name, trademark, manufacturer, or otherwise, does not necessarily constitute or imply its endorsement, recommendation, or favoring by the United States Government or the University of California. The views and opinions of authors expressed herein do not necessarily state or reflect those of the United States Government or the University of California, and shall not be used for advertising or product endorsement purposes.

This work was performed under the auspices of the U.S. Department of Energy by University of California, Lawrence Livermore National Laboratory under Contract W-7405-Eng-48. 


\title{
$\beta$-decay experiments and the unitarity of the CKM matrix
}

\author{
P.E. Garrett \\ Lawrence Livermore National Laboratory, PO Box 808, Livermore, California, 94551, United States
}

\begin{abstract}
The goal of this project was to perform very precise measurements of super-allowed Fermi $\beta$ decay in order to investigate a possible non-unitarity in the CKM matrix of the Standard Model of particle physics. Current data from 9 precisely measured $\beta$ decays indicated that the sum-of-squares of the first row of the CKM matrix differs from 1.0 at the $2.2 \sigma$ (or $98 \%$ confidence) level. If true, it would be the first firm indication of physics beyond the Standard Model - the model that has been the backbone of the worldwide physics community for more than 30 years. The physics goal of the project was to test and constrain the calculated correction factors that must be applied to the experimental data by performing measurements at the TRIUMF radioactive ion beam facility ISAC. Accurate and precise (precision goal $>99.9 \%$ ) half lives and decay branching ratios were measured for nuclei where different sets of calculated corrections give divergent results thereby allowing us to determine which theory, if any, gives the correct result. The LLNL contribution was to design and build the data acquisition system that will enable the experiments, and to provide theoretical calculations necessary for the interpretation of the results. The first planned measurement was ${ }^{34} \mathrm{Ar}$, to be followed by ${ }^{62} \mathrm{Ga}$ and ${ }^{74} \mathrm{Rb}$. However, there were major problems in creating a suitable, intense beam of radioactive ${ }^{34} \mathrm{Ar}$. The collaboration decided to proceed with measurements on ${ }^{62} \mathrm{Ga}$ and ${ }^{18} \mathrm{Ne}$. These experiments were performed in a series of measurements in the summer and fall of 2004. The LLNL team also is leading the effort to perform measurements on ${ }^{66} \mathrm{As}$ and ${ }^{70} \mathrm{Br}$ that are expected during $2006-2008$. While the definitive experiments to meet the goals of the LDRD were not conducted during the funding period, the involvement in the radioactive program at TRIUMF has lead to a number of new initiatives, and has attracted new staff to LLNL. This LDRD has laid the foundation for involvement in one of the world-leading radioactive beam facilities.
\end{abstract}

\section{INTRODUCTION}

The unification of the electromagnetic force and the weak nuclear force, which are two of the four basic forces of nature, by the Standard Model of electroweak interactions has been one of the most successful and important scientific breakthroughs of the 20'th century. The Standard Model remains unscathed by many experimental attempts over the past 30 years to find physics that would require its extension. These experiments range from the domain of high-energy physics, where B-factories like those at the Stanford Linear Accelerator are investigating the heavy quark and lepton sectors, to the domain of lowenergy nuclear physics. While the Standard Model has passed all experimental tests, many think it is not the "final" theory since it has many parameters that must be determined from experiment rather than predicted by the theory.

There is evidence from precise $\beta$-decay experiments that the CKM matrix (defined below), which plays a central role in the Standard Model, may not satisfy unitarity. Doubts on this result are cast, however, because of the calculated correction factors that must be applied to the data. The goal of the LDRD was to test these correction factors by performing measurements in systems where different calculations yield significantly different results. The LLNL team was invited into the collaboration for its expertise in data acquisition and experience in making precision measurements. It quickly assumed a leading role in defining how the experiments would be performed by providing the precision "clock" that enabled high-precision half-life measurements. In addition, the data-acquisition system 
LDRD Final Report 02- $L W-026$

was upgraded by the LLNL team into a worldclass system capable of high throughput and the ultimate in high precision.

\section{SCIENTIFIC MOTIVATION: SUPER- ALLOWED FERMI $\beta$-DECAY}

The physics being addressed with the $8 \pi$ spectrometer is quite varied, but here only the super-allowed Fermi $\beta$ decay programme is highlighted. This programme aims to perform detailed tests of the Standard Model of particle physics in the electroweak sector. Performing very precise measurements on nuclei that undergo the process of super-allowed Fermi $\beta$ decay tests the unitarity of the CabbiboKobayashi-Maskawa (CKM) matrix.

The basic building blocks of the Standard Model rest on three generations or families of particles, each family consisting of 2 quarks and 2 leptons. Leptons, like the electron and the neutrino, do not participate in the strong nuclear force, the interaction responsible for assembling quarks into nucleons and binding of nucleons into nuclei. The generations are:

$$
\begin{aligned}
& \left(\begin{array}{l}
u \\
d
\end{array}\right) \quad\left(\begin{array}{l}
c \\
s
\end{array}\right) \quad\left(\begin{array}{l}
t \\
b
\end{array}\right) \\
& \left(\begin{array}{c}
\boldsymbol{v}_{e} \\
\boldsymbol{e}
\end{array}\right) \quad\left(\begin{array}{c}
\boldsymbol{v}_{\mu} \\
\boldsymbol{\mu}
\end{array}\right) \quad\left(\begin{array}{c}
\boldsymbol{v}_{\boldsymbol{\tau}} \\
\boldsymbol{\tau}
\end{array}\right)
\end{aligned}
$$

where the $u, d, c, s, t$, and $b$ are the up, down, charm, strange, top, and bottom quarks, respectively, and the $e, \mu$, and $\tau$ are the electron, muon, and tau particles, with their associated neutrinos $v$. Only the quarks participate in the strong interaction, all family members (quarks and leptons) participate in the electroweak interaction.

Because of the electroweak interaction, the quark mass eigenstates are not the electroweak eigenstates. A change of basis is therefore needed, which is essentially a rotation of the basis vectors from the strong basis into the electroweak one. This is accomplished by using the CKM matrix that is written as:

$$
\left(\begin{array}{c}
d^{\prime} \\
s^{\prime} \\
b^{\prime}
\end{array}\right)=\left(\begin{array}{lll}
V_{u d} & V_{u s} & V_{u b} \\
V_{c d} & V_{c s} & V_{c b} \\
V_{t d} & V_{t s} & V_{t b}
\end{array}\right)\left(\begin{array}{l}
d \\
s \\
b
\end{array}\right)
$$

and performs the transformation. The CKM matrix must be unitary, i.e., the sum of the squared matrix elements in a particular row or column must be equal to 1 . New physics is required if it is found that a row in the CKM matrix does not obey unitarity, regardless of the level of violation. Some possibilities for new physics are the presence of right-handed currents (to date, only left-handed currents are known to participate in the weak interaction), in-medium renormalization of the quark masses, or scalar interactions [1].

The first row of the CKM matrix is the only one that can be tested presently for unitarity with sufficient precision. The values of the matrix elements [2] for the first row are $V_{u d}=0.9738 \pm 0.004 V_{u s}=0.2200 \pm 0.0026$, and $V_{u b}=0.0036 \pm 0.0007$ leading to:

$$
V_{u d}^{2}+V_{u s}^{2}+V_{u b}^{2}=0.9965 \pm 0.0014
$$

that differs from unitarity at the $98 \%$ confidence level [2]. While the difference from the unitarity value may appear small, $0.9965(14)$ as compared to unity, this small difference has truly profound consequences.

The matrix element $V_{u d}$ is the largest term in the above squared sum, and also has the largest uncertainty. It can be derived from super-allowed $0^{+} \rightarrow 0^{+}$Fermi $\beta$ decays between isobaric analogue states [2], e.g. ${ }^{14} \mathrm{O}$ $\rightarrow{ }^{14} \mathrm{~N}$. By restricting to decays occurring between $0^{+}$states, only the vector current, $V$ of the $V-A$ weak interaction, contributes to the decay process. The rate at which these decays occur can be related to the $V_{u d}$ matrix element via the weak-vector coupling constant $G_{V}$. According to the Standard Model, $V_{u d}$ is given by:

$$
V_{u d}^{2}=\frac{G_{V}^{2}}{G_{F}^{2}}
$$

where $G_{F}^{2}$ is the Fermi coupling constant obtained from muon decay (and known very precisely [2]). The factor $G_{V}^{2}$ is determined from Fermi super-allowed $\beta$ decay between isospin $T=1$ multiplets via the expression: 


$$
F t=\frac{K}{2 G_{V}^{2}\left(1+\Delta_{R}\right)}
$$

where $K /(\hbar c)^{6}=(8120.271 \pm 0.012) \times 10^{-10}$ $\mathrm{GeV}^{-4} \mathrm{~S}$ is a constant, and $\_R=(2.40 \pm 0.08) \%$ is a nucleus-independent radiative correction. The quantity $F t$ is what is determined in the $\beta$ decay process, where $t$ is the $0^{+} \rightarrow 0^{+}$partial half-life $t=t \times B R$ with $B R$ the fraction of decays that proceed via the $0^{+} \rightarrow 0^{+}$decay path versus all decay paths. $F$ is not measured directly, but is related to the Fermi factor $f$ via [2]

$$
F=f\left(1-\delta_{C}\right)\left(1+\delta_{R}\right) \text {. }
$$

The Fermi factor $f$ takes into account: 1) phase space effects, and 2) the Coulomb interaction of the $\beta$ particles with the nucleus. Two small corrections have to be applied; a radiative correction, $\delta_{R}$, and a nuclear structure correction, $\delta_{C}$ due to isospin-symmetry breaking. According to the Standard Model, the $F t$ values for super-allowed Fermi $\beta$ decay should be a constant (the conserved-vectorcurrent hypothesis), and any deviation from this would herald new physics.

To date, the data are precise enough for only 12 nuclei to be included in the extraction of $V_{u d}$, although the uncertainties on 3 of these are much larger than the other 9 and so have practically no impact [2]. Shown in Fig. 1 is the current status of the data for the nuclei used. It is important to note that the error associated with $\left|\mathrm{V}_{\mathrm{ud}}\right|$ is not predominantly experimental in origin; the largest uncertainties come from $\Delta_{R}( \pm 0.0004)$ and $\delta_{C}( \pm 0.0003)$. Also shown on the plot are the shifts in the data that would need to be applied in order to satisfy the unitarity condition.

The value of $V_{u d}$ can also be determined from the neutron and pion beta decay. These decays have the advantage over the superallowed Fermi decay of the nucleus that there are no nuclear structure-dependent corrections. A recent measurement [3] of the neutron decay asymmetry results in the value of $V_{u d}$ that is $\sim 3$ times less precise than that from nuclear decays, and leads to an even smaller unitarity sum. A new measurement (PIBETA) of a very weak $\left(\sim 10^{-8}\right)$ pion decay branch is underway at the Paul Scherrer Institute in Switzerland. This could lead to a factor of seven improvement in precision [6], however at the present time the value of $\left|V_{u d}\right|$ obtained from pion decay agrees with that obtained from super-allowed Fermi decay but is a factor of ten less precise. Ultimately, both decays will also be limited by the theoretical uncertainty in $\Delta_{R}$.

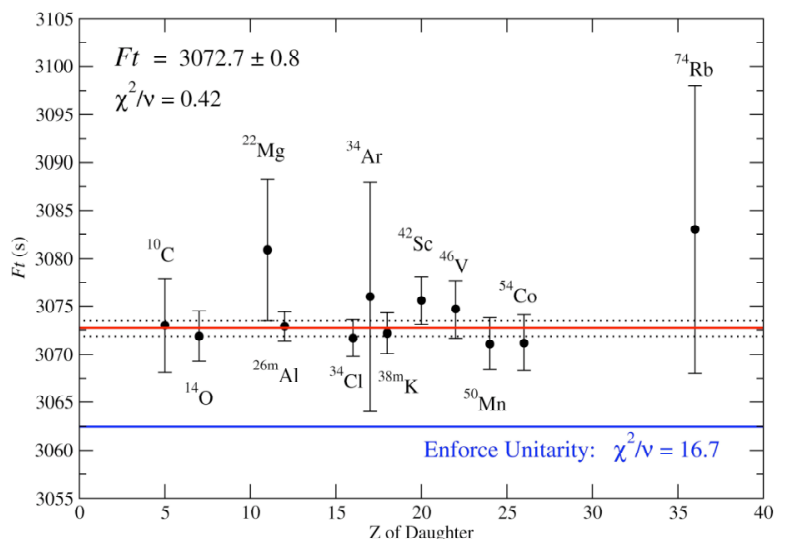

Figure 1: Values of $F t=f t\left(1-\delta_{C}\right)\left(1+\delta_{R}\right)$ for the nuclei measured with sufficient precision to be included in the CKM unitarity test.

Recently, attention has focused on the value of $V_{u s}$. The $E 865$ experiment at Brookhaven recently reported [4] a new value of $V_{u s}$ from a high statistics measurement of $K_{e 3}^{+}$decay. The result, $0.2272 \pm 0.0023_{\text {stat }}$ $\pm 0.0019_{\text {f }}$, disagrees with the value recommended by the Particle Data Group [5], and would eliminate the unitarity problem. However, it is also inconsistent with preliminary results from the $K L O E$ experiment that is able to measure all four decay branches from the semileptonic decay of neutral and charged kaons, i.e. $K_{e 3}^{+}, K_{\mu 3}^{+}, K_{e 3}^{0}$ and $K_{\mu 3}^{0}$ branching ratios [6]. In these measurements it is necessary to determine not only the decay rate but also the momentum dependence of two form factors as well as theoretical estimates of isospin and $\mathrm{SU}(3)$ symmetry breaking effects. The KteV (E832) experiment at Fermilab recently reported [7] a value for $V_{u s}=0.2252(22)$. Cabibbo [8], from a reanalysis of semileptonic hyperon decays neglecting SU(3) symmetry breaking effects, obtained a value of $V_{u s}=0.2250(27)$. In contrast, the $N A 48$ collaboration at CERN 
found a value of $V_{u s}=0.2187(22)$ [9], in agreement with previous measurements. In this case, the uncertainty was dominated by the theoretical uncertainty in the form factor, $f_{+}(0)$. With a number of new experiments in progress (E865, KLOE, NA48/2, and CMD2) [6], the present uncertainty in the value of $V_{u s}$ is expected to be resolved in the coming years. Nevertheless, continued effort to reduce the uncertainty in $V_{u d}$ remains a high priority since the unitarity test of the elements in the first row of the CKM matrix is, at the present time, the most precise unitarity test that can be applied to the CKM matrix.

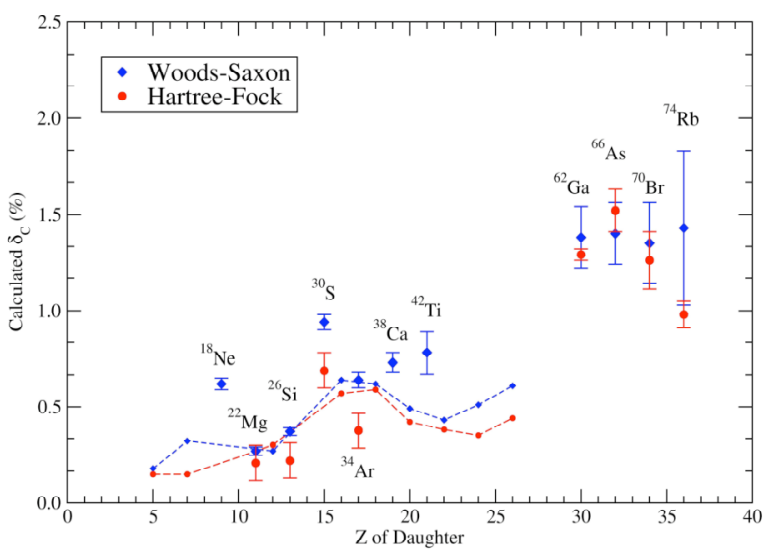

Figure 2: Calculated isospin-symmetry-breaking corrections $\delta_{C}$ for super-allowed Fermi $\beta$ decays. Shown are two different approaches to calculate the corrections from Woods-Saxon calculations (TownerHardy) and Hartree-Fock (Ormand-Brown) calculations. Points connected by lines are for the calculated values for the nine precisely known cases. The differences between the two calculations for these cases are relatively small. For the remaining nuclei, the differences can be quite large, approaching a factor of nearly 2 for ${ }^{34} \mathrm{Ar}$.

Since the failure in the unitarity of the CKM matrix would imply physics beyond the minimal Standard Model, it is important to eliminate all possible "trivial" explanations for the apparent discrepancy. The leading order terms in the radiative corrections, $-R$ and $\delta_{R}$, are based on quantum electrodynamics and hence it is believed that they cannot be responsible for the dramatic shift that would be required to satisfy unitarity $[1,2,10]$. The nuclear-structure-dependent Coulomb correction $\delta_{C}$ is less well known. There are two "standard" calculations [1,2,10-13] for the Coulomb corrections. For the nine precisely measured $f t$ values that are currently used in the determination of CKM unitarity, the differences between the two calculations of $\delta_{C}$ are small, although there is a systematic difference of $\approx 0.07 \%$ between them. These points are shown in Fig. 2 connected by lines. For other nuclei, such as ${ }^{34} \mathrm{Ar}$ or ${ }^{74} \mathrm{Rb}$, there can be very large differences. Because of these differences, a systematic uncertainty is introduced that is one of the largest contributions to the $F t$ value. One of the prime goals of the ongoing experimental program at TRIUMF is to test these correction factors by performing precision measurements on nuclei where there are substantial differences between the two competing calculations. Improvements will come as the number of super-allowed emitters accessible to precision measurements increases. In particular, the $\delta_{C}$ corrections are predicted to be much larger for the heavier $f p$-shell nuclei [13]. It was the purpose of the LDRD to test these correction factors by performing precision measurements on nuclei where there are substantial differences between the two competing calculations.

According to the Standard Model, the Ft values extracted from any one super-allowed Fermi $\beta$ decay must agree with all other extracted $F t$ values. Therefore, in principle we can test which calculation gives the correct $F t$ value if we have accurate measurements on those nuclei where there are large differences in the calculated correction factors. Once we settle the question of which calculation provides the proper answer, or indicate weaknesses in both calculations, progress on the question of CKM unitarity can be made. It has to be emphasized here that a difference in the overall correction on the order of $0.2 \%$ is all that is needed to satisfy unitarity. It is therefore critical that the nuclear-structure dependent corrections be examined thoroughly. The only way to accomplish this to obtain additional high-quality data on those nuclei where the differences between the calculations are large. A further goal of this LDRD was to examine the possible $Z$ or $A$ dependence in the extracted $F t$ values. The $\mathrm{G}_{\mathrm{V}}{ }^{2}$ value determined from the intercept of the 
LDRD Final Report 02-LW-026

first order linear fit to the $F t$ values is consistent with unitarity of the CKM matrix at the $1 \sigma$ level. In order to test this dependence, data on high-mass superallowed Fermi $\beta$ decay must be obtained. The LLNL team is leading this effort, with a successful proposal submitted to the TRIUMF Experiment Evaluation Committee in Dec. 2003 to examine the decay of ${ }^{66}$ As and ${ }^{70} \mathrm{Br}$.

Substantial progress has already been made in studying these decays. The half life and branching ratio for ${ }^{74} \mathrm{Rb}$ decay have been determined [14,15], and further mass measurements are needed. The observed strength for all non-superallowed $\beta$ decays of ${ }^{74} \mathrm{Rb}$ was determined to be $0.5(1) \%$. Note that this requires only a $20 \%$ relative measurement, and is characteristic of the heavier Fermi super-allowed decays. Measurements on ${ }^{62} \mathrm{Ga}$ decay have also been made $[16,17]$. In addition to these, a study [18] of ${ }^{18} \mathrm{Ne}$ has been made, although in this case the measurement of the branching ratio to the required precision will be extremely difficult since the super-allowed branch is only $7.7 \%$. Also planned are experiments on ${ }^{34} \mathrm{Ar}$, a remeasurement of the ${ }^{62} \mathrm{Ga}$ lifetime and the ${ }^{74} \mathrm{Rb}$ branching ratio, and a series of measurements on ${ }^{66} \mathrm{As}$ and ${ }^{70} \mathrm{Br}$.

\section{EXPERIMENTAL APPROACH}

A unique opportunity for study of superallowed Fermi $\beta$ decay exists using the radioactive ion-beam facility ISAC at TRIUMF (Vancouver, Canada). The LLNL team used its expertise in experimental techniques to enable the accurate half life and branching ratio measurements to test CKM

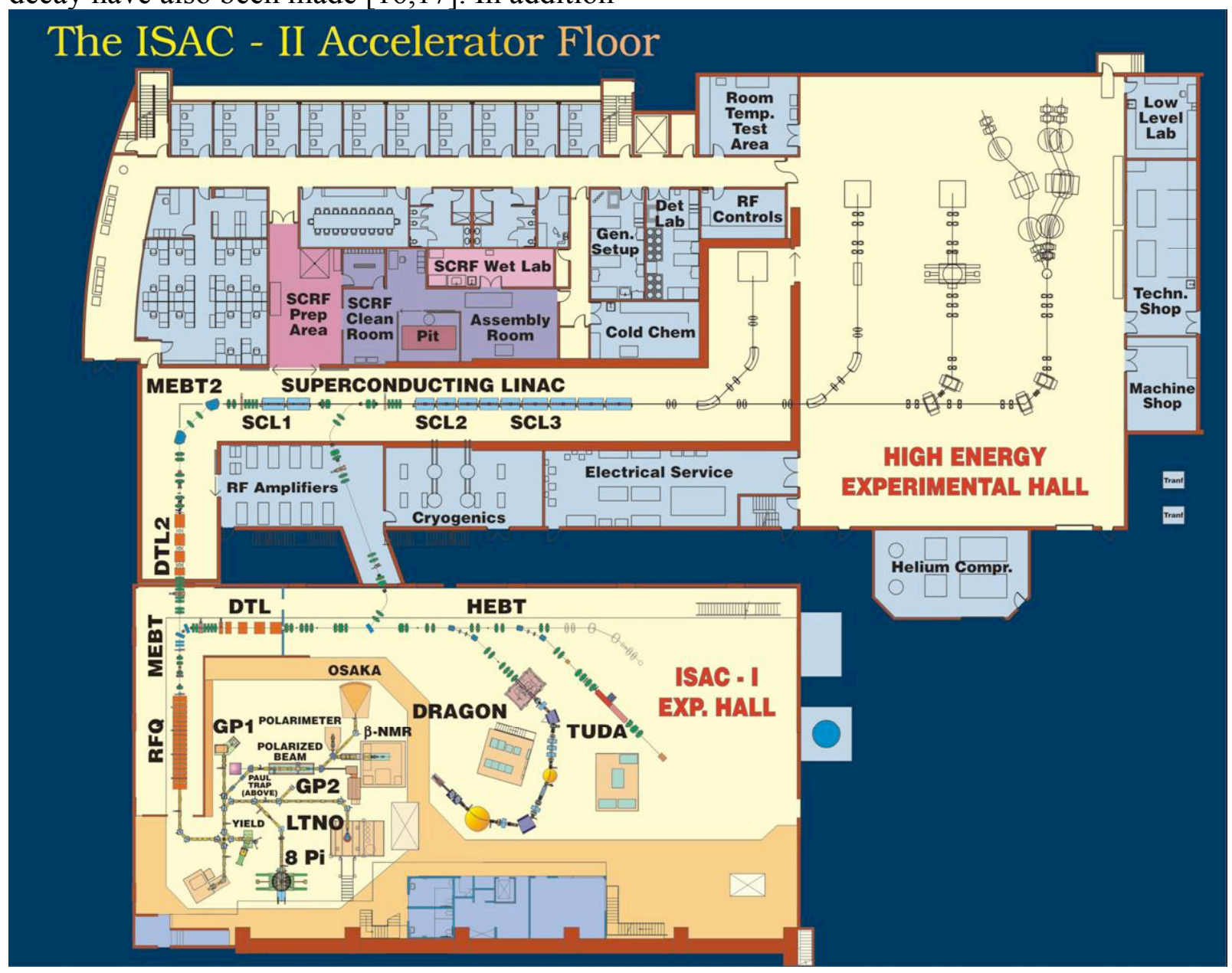

Figure 3. Physical layout of the TRIUMF ISAC and ISAC-II facilities. ISAC is capable of delivering beams of any mass (produced by the target) with an accelerating potential of up to $60 \mathrm{KV}$, and accelerating beams up to mass 30 and $\sim 1.5 \mathrm{MeV} /$ nucleon. ISAC-II currently in construction will extend the accelerated beams up to mass $\sim 150$ and $\sim 6.5 \mathrm{MeV} /$ nucleon. 
LDRD Final Report 02-LW-026

unitarity. We were and are an integral part of an international collaboration using the TRIUMF facility to study CKM matrix unitarity, and we played and continue to play a leading role in these experiments. The ISAC facility at TRIUMF is an ISOL-type (Isotope Separator On-Line) facility that employs a 500 $\mathrm{MeV}$ proton beam impinging on a spallation target. Various spallation targets have been used, including $\mathrm{Ta}, \mathrm{CaZrO}_{3}, \mathrm{Nb}, \mathrm{SiC}$, and $\mathrm{ZrC}$. Proton beam currents of up to $70 \mu \mathrm{A}$ on a specially designed high-power Ta target have been used. Plans to develop $\mathrm{LaC}$ and UC targets are in place that will extend the reach of ISAC to neutron-rich radioactive beams. Measured yields of various radioactive beams are provided on the web-site http://www.triumf.ca/people/marik/Yields.html.

To date, beams have been produced with a surface ion source, an ECR source, and a laser ion source. New ion sources under development are FEBIAD and negative-ion sources. Figure 3 shows the physical layout of the ISAC and ISAC-II buildings and some of the installed experiments. ISAC is capable of delivering beams of any mass with an accelerating potential of up to $60 \mathrm{kV}$ (although usually $30 \mathrm{kV}$ is used), and accelerated beams up to mass 30 and energies of $\sim 1.5$ $\mathrm{MeV} /$ nucleon. ISAC-II, currently under construction, is designed to accelerate beams up to mass 150 and energies of $\sim 6.5$ $\mathrm{MeV} /$ nucleon. Further information on these facilities can be found at http://www.triumf.ca.

\section{INSTRUMENTAL CAPABILITIES}

The program at TRIUMF focuses on precision half life and branching ratio determination. The primary tool for these investigations is the re-built and re-configured $8 \pi$ spectrometer, an array of $20 \mathrm{hpGe}$ detectors for $\gamma$-ray detection. Complementing this is an array of 20 plastic scintillator detectors (SCEPTAR) for positrons for $\beta-\gamma$ coincidence studies. The initial LLNL contribution was the construction of a precision "clock", with a precision on the order of $10^{-8}$, to accurately measure the half lives of the $\gamma$ rays associated with the decay. Our role in the collaboration greatly expanded; we designed and assembled the front-end of the data acquisition system.

In the first year of this LDRD, we performed an assessment of the adequacy of the data acquisition system then in use, and found that it was not capable of delivering the reliability and flexibility needed to enable the precision measurements. Major upgrades in capabilities were needed. It was in this arena that we took a leading role; our experience in high-precision high-throughput data acquisition was unique to the collaboration.

The $8 \pi$ spectrometer currently comprises 4 different detector systems; Comptonsuppressed Ge detectors (the $8 \pi$ spectrometer) for $\gamma$-ray detection, plastic scintillators (named the SCintillating Electron Positron Tagging ARray - SCEPTAR) for detection of $\beta$ particles, $\mathrm{BaF}_{2}$ detectors (named the Dipentagonal Array for Nuclear Timing Experiments - DANTE) for $\gamma$-ray detection with fast-timing measurements, and $\mathrm{Si}(\mathrm{Li})$ detectors (named the Pentagonal Array for Conversion Electron Spectroscopy - PACES) for conversion electron studies. An integral part of the spectrometer is the Moving Tape Collector (MTC), and control over beam pulsing and tape cycling.

\section{A. The $8 \pi$ Spectrometer}

The $8 \pi$ spectrometer was originally designed for high $\gamma$-ray multiplicity studies of nuclei excited in heavy-ion fusion evaporation reactions, and consisted of 20 Comptonsuppressed Ge detectors with a 70-element BGO calorimeter. The basic geometry of the spectrometer is based on the truncated icosahedron that is a 32-faced tiling of a sphere corresponding to the facial arrangement of 20 hexagonal and 12 pentagonal shapes. (It is the shape used in the construction of most soccer balls.) The positions of the Ge detectors, Ortec HPGe with a nominal relative efficiency of $\sim 25 \%$, occupy the 20 hexagonal positions, with 4 rings of 5 detectors at angles of $\pm 37^{\circ}$ and $\pm 79^{\circ}$ with respect to the beam direction. 


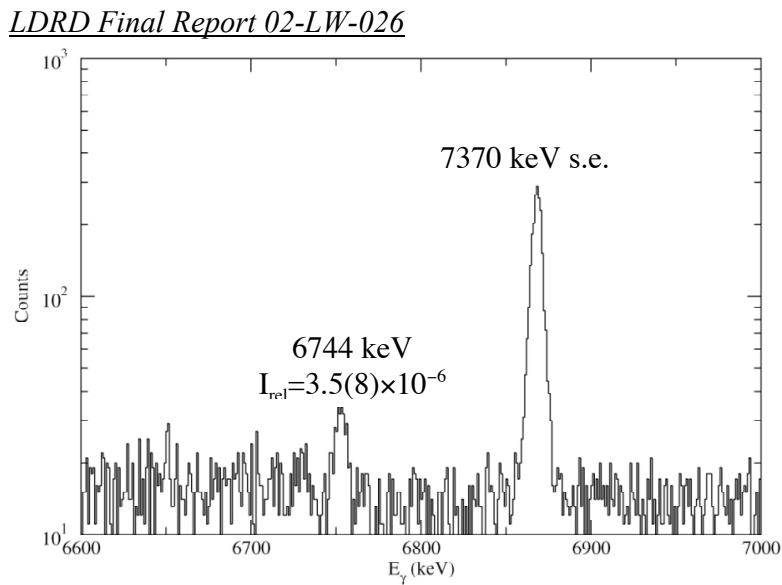

Figure 4: Portion of the $\gamma$-ray spectrum observed in the decay of ${ }^{26} \mathrm{Na}$. The radioactive beam intensity was $\sim 10^{6}$ ions/s and data was collected for 10 hours duration in $\gamma$ ray singles mode.

With the removal of the 70-element inner BGO ball, the Ge detectors and their BGO Compton shields were moved inwards in order to improve detection efficiency. The target-tofront-face distance for the BGO suppression shields is now $13 \mathrm{~cm}$, and that for the $\mathrm{Ge}$ detectors is $14 \mathrm{~cm}$. Two different sets of collimators can be used: $2 \mathrm{~cm}$ thick densalloy collimators to prevent the BGO shields and the outer radius of the coaxial Ge detectors from the direct irradiation from the source, or a $1 \mathrm{~cm}$ thick densalloy collimator with a $1 \mathrm{~cm}$ thick delrin shield can be installed to attenuate highenergy $\beta$ particles that may reach the Ge detectors. The absolute photopeak efficiency of the reconfigured array has been measured for the $1332-\mathrm{keV}^{60} \mathrm{Co}$ line to be $1.5 \%$, with a peak-to-total ratio of 0.41 . To demonstrate the sensitivity of the reconfigured $8 \pi$ array, shown in Fig. 4 are results from an experiment [19] using a ${ }^{26} \mathrm{Na}$ beam of $10^{6}$ ions/s of 10 -hour duration. A sensitivity on the relative $\beta$-decay branching on the order of $10^{-6}$ was achieved.

\section{B. SCEPTAR}

Complementing the Ge detectors is the SCEPTAR array of 20 plastic scintillators that are arranged into 2 rings of 5 trapezoidal pieces and 2 rings of 5 rectangular pieces. The positioning is such that one plastic scintillator overlaps the solid angle of one Ge detector. The total solid angle coverage of SCEPTAR is approximately $80 \%$ of $4 \pi$. The plastic scintillators are BC410 of thickness $1.6 \mathrm{~mm}$. Light is collected from the edge of the scintillators and transported via $\sim 25 \mathrm{~cm}$ long light-guides to the phototubes located outside of the main frame of the array. Figure 5 is a photo showing portions of the $8 \pi$ and SCEPTAR arrays. As an example of the power of SCEPTAR-Ge coincidences to extract weak signals from background, shown in Fig. 6 are spectra obtained in a preliminary experiment to study the level scheme of ${ }^{32} \mathrm{Mg}$ via ${ }^{32} \mathrm{Na}$ decay. The ${ }^{32} \mathrm{Na}$ beam intensity, obtained with a $40 \mu \mathrm{A}$ primary beam on a Ta target, was only $\sim 2$ ions/s. The top panel displays the spectrum obtained using only the $\gamma$-ray singles condition, whereas the spectrum in the lower panel results from the requirement of coincidences with $\beta$-particles detected in SCEPTAR. While the ${ }^{32} \mathrm{Mg}$ lines are invisible in the ungated spectrum, they become the most prominent feature in the coincidence spectrum - thus demonstrating that spectroscopy is possible with beam intensities as low as a few ions/s. This nucleus will be revisited once the planned UC target is available. The use of an UC target will boost the yield of ${ }^{32} \mathrm{Na}$ by several orders of magnitude.

\section{PACES}

The most recent addition to the arsenal of detectors is PACES. An array of $5 \mathrm{Si}(\mathrm{Li})$ detectors, PACES makes available both conversion-electron and internal-pair spectroscopy, the latter of which is advantageous far from stability where the Qvalue is large. Inclusion of conversionelectron data provides not only multipolarity information, but also reveals electric monopole (EO) transitions indicative of shape coexistence. The $\mathrm{Si}(\mathrm{Li})$ detectors are approximately $5 \mathrm{~mm}$ in thickness and have a typical resolution of $2.5 \mathrm{keV}$ at $1 \mathrm{MeV}$. A close-up view of the PACES array is shown in Fig. 7. 


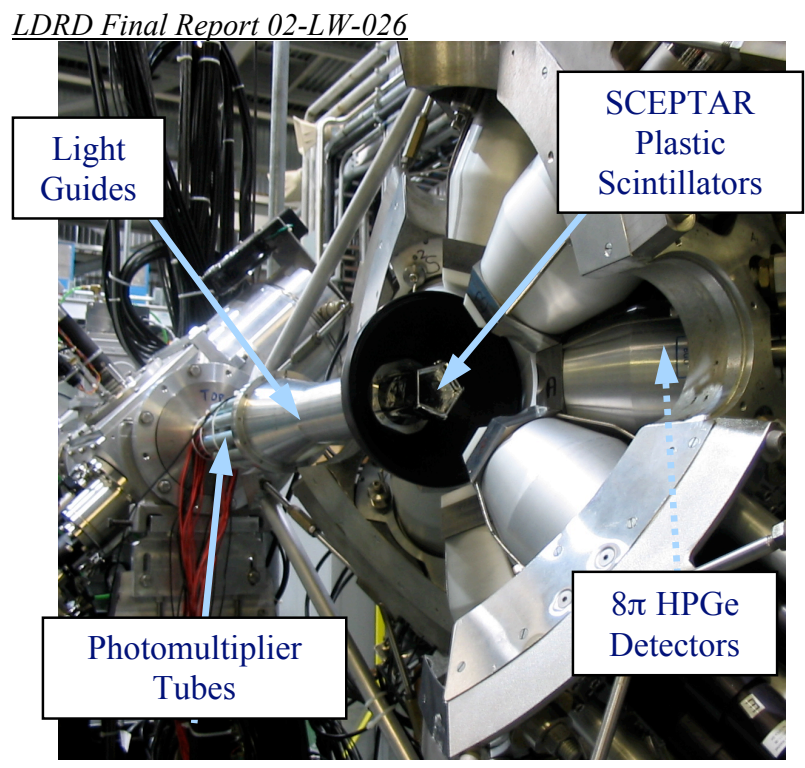

Figure 5: Photograph of the upstream portion of the SCEPTAR plastic scintillator array and one hemisphere of the $8 \pi$ array.

The conversion-electron data from PACES are vital for the establishment of multipolarity of transitions. Furthermore, in some lowenergy or isomeric decays, the decaying transitions are not observed because of their large conversion coefficient. A very recent example is provided by the decay of a new isomeric state found in ${ }^{174} \mathrm{Tm}$ [20]. Originally favoured to be a high $K$ isomer, knowledge of the multipolarity of the transitions leads to the assignment [21] of $0^{+}$for the isomeric state.

\section{DANTE}

The detector system DANTE is an LLNLled proposal to enable very-fast lifetime measurements following $\beta$ decay. Using the available 10 open pentagonal positions in the $8 \pi$ spectrometer (1 pentagon is used for the incoming beam line, and another for the vacuum system for the moving tape collector and SCEPTAR light guides), the $10 \mathrm{BaF}_{2}$ detectors of DANTE are being installed. The $\mathrm{BaF}_{2}$ scintillator is the fastest known; one of its two scintillating-light components has a decay constant of $0.6 \mathrm{~ns}$ and emits light in the UV region. The $\mathrm{BaF}_{2}$ detectors, purchased by the University of Guelph (Canada), were obtained from SCIONIX and are in the shape of a truncated cone $2 \mathrm{~cm}$ diameter at the top, $4 \mathrm{~cm}$ at the bottom, and $3 \mathrm{~cm}$ in length on top of a

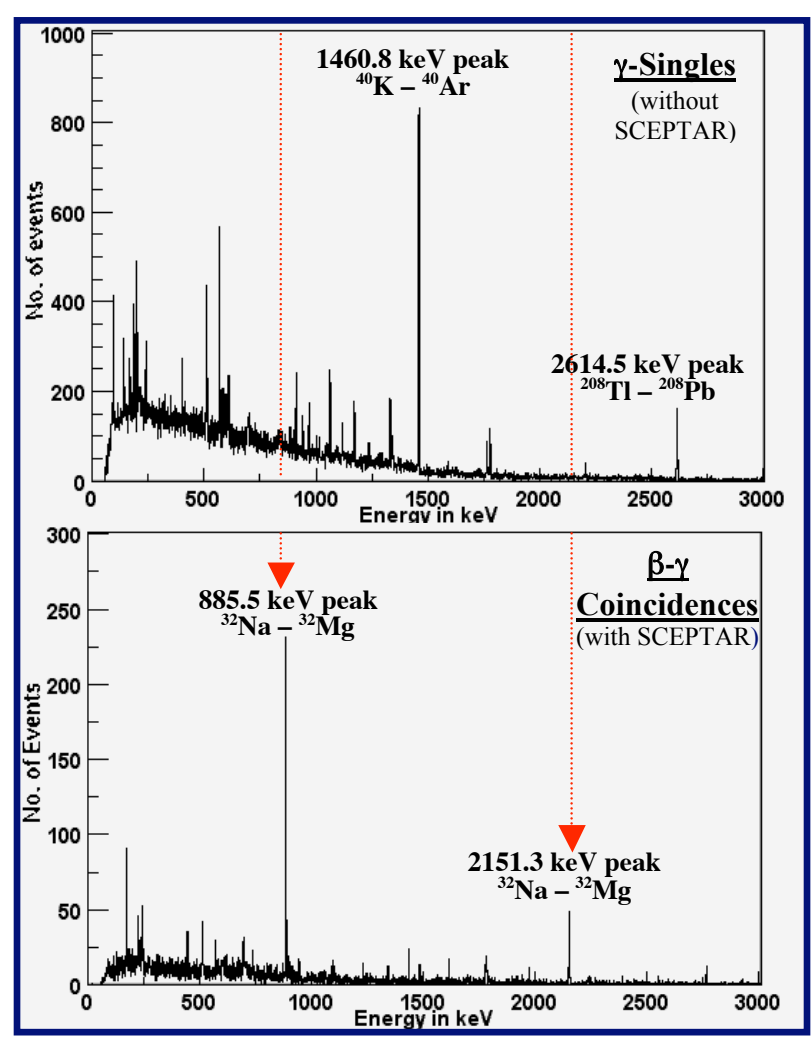

Figure 6: Results of a preliminary experiment with a ${ }^{32} \mathrm{Na}$ beam of $\sim 2$ ions/s. In the top spectrum, the ${ }^{32} \mathrm{Mg}$ lines are invisible due to the natural background, whereas by requiring coincidences with the $\beta$-particles detected with SCEPTAR, the ${ }^{32} \mathrm{Mg}$ lines become the most prominent in the spectrum.

cylinder of diameter $4 \mathrm{~cm}$ and $1.7 \mathrm{~cm}$ in length. The detectors are coupled to very fast photomultiplier tubes, the Photonis XP2020/URQ, having a quartz entrance window for maximum transmission of the fastcomponent UV light. An initial test experiment to accurately map the time response of the plastic scintillators and test the feasibility of the DANTE array used $4 \mathrm{BaF}_{2}$ detectors from the University of Surrey. Using a ${ }^{26} \mathrm{Na}$ radioactive beam, it was found that the individual SCEPTAR detectors had timing resolutions (FWHM) in the range of $1-1.5 \mathrm{~ns}$. With $\mathrm{BaF}_{2}-\mathrm{BaF}_{2}$ coincidences, on the other hand, the timing FWHM of $\approx 200$ ps was ultimately achieved. Figure 8 displays spectra collected during the test experiment. In panel a), results from a ${ }^{152} \mathrm{Eu}$ calibration source are shown, where the lifetime of the $122-\mathrm{keV}$, $\tau=1.3 \mathrm{~ns}, 2^{+} \rightarrow 0^{+}$transition can be easily extracted. In panel $b$ ), the pulse height spectrum obtained with a ${ }^{26} \mathrm{Na}$ beam displayed, 


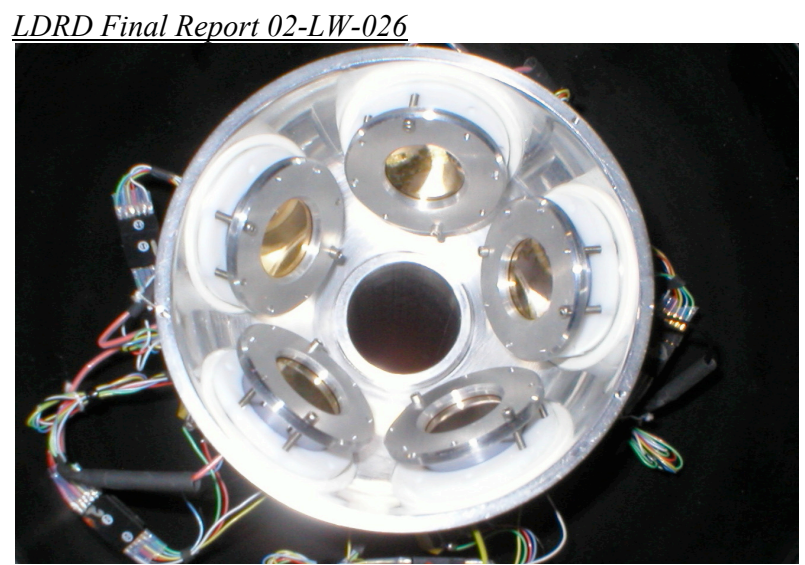

Figure 7: The PACES array of $5 \mathrm{Si}(\mathrm{Li})$ detectors for conversion-electron spectroscopy. The $\mathrm{Si}(\mathrm{Li})$ detectors subtend $\sim 5 \%$ of the $4 \pi$ solid angle.

with the time spectrum for plastic- $\mathrm{BaF}_{2}$ coincidences and $\mathrm{BaF}_{2}-\mathrm{BaF}_{2}$ coincidences shown in the inset. Finally, panels c) and d) are results using the $\mathrm{BGO}$ Compton suppression shields of the Ge detectors to form an anti-Compton shield for the $\mathrm{BaF}_{2}$ detectors, with c) the accepted spectrum and d) the rejected spectrum. The improvement in quality of the spectrum using this Comptonrejection scheme is notable. This test represents the first time (to our knowledge) that timing $\mathrm{BaF}_{2}$ detectors have been Compton suppressed. This will lead to a significant reduction in the systematic uncertainty assigned to the short level lifetimes, since one of the dominating contributions arises from the Compton distribution under the peak.

\section{E. Data Acquisition System}

In order to achieve the precision needed for the super-allowed decay studies, required to be better than $0.1 \%$, a high degree of diagnostics are required and every event must be accounted for. Further, since one of the prime experiments is the determination of the ${ }^{34} \mathrm{Ar}$ half-life by $\gamma$-ray measurements, extreme precision must be accomplished with the $\gamma$-ray detectors. The presence of additional auxiliary detectors could influence the performance of data acquisition of the $\gamma$-ray data. Therefore, the approach adopted was to decouple as far as possible the acquisition of data from the various detectors. This is achieved by having for each detector system its own "data stream", with its own trigger and time stamping of all
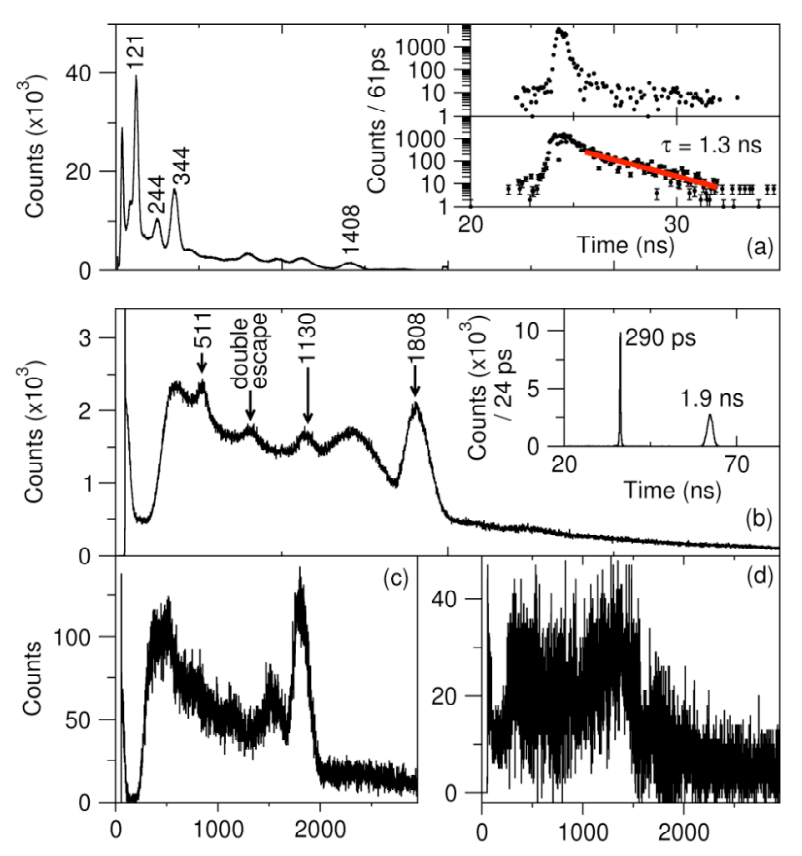

Figure 8: Results from an LLNL-led test run using 4 $\mathrm{BaF}_{2}$ detectors. The spectra in panel a) are obtained with a ${ }^{152} \mathrm{Eu}$ calibration source, and display the ease with which lifetimes on the order of a ns can be extracted. The spectra in panel b) are results with the ${ }^{26} \mathrm{Na}$ beam displaying $\mathrm{BaF}_{2}-\mathrm{BaF}_{2}$ timing of 290 ps FWHM and $\mathrm{BaF}_{2}$-SCEPTAR timing of $1.9 \mathrm{~ns}$. When optimized for $\mathrm{BaF}_{2}-\mathrm{BaF}_{2}$ timing, a resolution of $\sim 200$ ps was achieved. Panels c) and d) display the Comptonsuppressed and rejected spectra employing the BGO shields of the surrounding Ge detectors.

events. These data streams can then be merged in software afterwards, and the appropriate correlations taken.

The previous data acquisition system was centered on CAMAC modules with CAMAC data transfer. Normally CAMAC data transfer is a rather slow process, achieving sustained speeds of about $1 \mathrm{MB} / \mathrm{s}$. It was recognized that much higher data-transfer rates would be necessary, due to 1) much higher counting rates that would be needed since many of the experiments would be conducted in "singles" mode, and 2) the increase in required diagnostic data words in each event increasing the event size, perhaps by as much as a factor of 2-3. While there are a number of dataacquisition standards that can easily achieve the much higher data-transfer rates necessary, many of these would require the purchase of an entirely new system. In an effort to implement the most cost-effective system, it 
LDRD Final Report 02-LW-026

was decided to use as many of the current modules as possible. The FERA based system, developed by LeCroy corporation in the 1980 's, remains a very robust and highlyreliable data-acquisition standard, and is capable of very high data-transfer rates of 200 ns per 16-bit data word, or $10 \mathrm{MB} / \mathrm{s}$.

As the $8 \pi$ spectrometer has expanded in the number of detector systems, the data acquisition has also expanded in its sophistication. Currently, it is composed of 4 separate data streams corresponding to the four different detector types; Ge, SCEPTAR, DANTE, and PACES. Each data stream is controlled by a Cheesecote Mountain CAMAC FERA driver, CMC203. The new FERA drivers were designed as a drop-in replacement for the older (now discontinued) LeCroy 4301 FERA driver, but incorporate a number of advancements and enhancements. The CMC203 has four operating modes; as a standard 4301 FERA driver, as a FERA driver but with a $2 \mathrm{k}$ FIFO between in input and output bus, as a FERA driver with a histogramming memory, and as a FERA driver with a $10^{6}$ word list-mode memory. With the latter two modes, the data are stored internal to the unit and must be read out via the CAMAC backplane (FASTCAMAC compatible), whereas in the first two modes the data can be read out either via the CAMAC backplane or via an ECL bus. The $8 \pi$ data acquisition uses the standard 4301 FERA-driver mode with data output on the ECL data bus. The different modes are selectable via control registers that can be written to via standard CAMAC commands. Also selectable via control registers are various other quantities such as the time between receiving the REQ signal and the generation of the read-out enable signal (REN), a time-out between receiving a GAI input (which is passed via the control bus to the other modules in the data stream and used as a Master-gate signal) and receiving a REQ, the length of the FERA clear signal, the time delay between the dropping of the REQ line and the end of the BUSY signal, etc. Data from the FERA driver is transferred to a Struck SIS3700 32 bit ECL FIFO memory unit located in a VME crate. Each data stream has its own memory unit. The memory units are polled for data, and upon the positive response of the event counter the data are readout from the data FIFO and transferred via TCP/IP to the data acquisition computer. The data are stored onto hard disk, and various histograms can be created for on-line analysis and monitoring.

\section{E.1 The Ge Data Stream}

For the Ge data stream, two identical signals from the Ge-detector preamplifiers are used; the first is fed into on Ortec 572 spectroscopy amplifier (hereafter labelled as the energy signal), while the second is used in the timing circuitry (timing signal). The energy signal from the amplifier typically has a Gaussian shaping time of $0.5-3 \mu \mathrm{s}$, depending on the application, and is fed directly from the amplifier into Ortec AD114 14-bit ADC's. The ADC's have both Master and Local gates applied; the Master gate originating from the Master Trigger for the Ge-stream, while the local gates are derived from the Ge-timing circuitry. The ADC's are setup via a control register, to yield zero-suppressed, nonoverflow suppressed, data that is available on the front ECL bus. The analogue part of the timing circuitry consists of timing-filter amplifiers, and Ortec 583b constant-fraction discriminators (CFD). The fast outputs from the CFD units are fed into LeCroy 4415 16channel discriminators, delayed approximately $128 \mathrm{~ns}$ and fanned-out using a LeCroy 4518 delay unit and then used as input into logic units (LeCroy 4516) for software-selectable Compton suppression. One of the outputs of the logic unit is used as input to a LeCroy 4532 majority logic unit (MALU). This unit is used in the ungated-unlatched mode with the majority level set to 1 , and thus it acts as an OR for the $20 \mathrm{Ge}$ detectors. The majority output from the MALU is used as the " $\gamma$ singles" input for subsequent trigger logic, and also as the start input for a LeCroy 222 Gate and delay generator that is set to approximately $1 \mu \mathrm{s}$ width. This latter signal is used as the gating signal for the $\gamma-\gamma$ coincidence circuitry. A second output of the Compton suppression logic unit is delayed approximately $100 \mathrm{~ns}$ and input into a second 
LDRD Final Report 02-LW-026

MALU operated in memory enabled mode that is gated with the $1 \mu \mathrm{s}$ wide $\gamma$-singles pulse. This MALU is self-cleared after the gate signal drops, and has its majority output level selected for coincidence of at least 2 inputs, forming the $\gamma$ - $\gamma$-coincidence signal for the trigger logic. The third output from the Compton-suppression logic unit is fed into a LeCroy 3377 multi-hit 32-channel TDC. The TDC is operated in common-stop double-word mode, and can accommodate up to 16 hits per channel. The stop or GAI signal for the TDC is derived from the Master Trigger for the Ge data stream, and it is delayed using a Stanford Research Systems DG535 precision delay generator by an amount approximately equal to the length of the master-gate signal applied to the Ortec ADC's.

The remaining units in the Ge data stream are 1) the BGO timing TDC; recording the time of the BGO hit relative to the Master Trigger, 2) the pileup TDC; the inhibit outputs from the linear amplifiers are converted and input to a TDC to be used as a pileup indicator with a time relative to the Master Trigger, and 3) a LeCroy 2367 Universal Logic Module (ULM); used in the latching scalar and bitregister mode. This final unit provides the key to the data acquisition system. Three 32-bit scalers are available in the programming of this unit; scaler 1 counts the number of pulses presented by a $10 \mathrm{MHz}$ high-precision pulser. The high-precision pulses are derived from a Stanford Research Systems $10 \mathrm{MHz}$ dualovenized oscillator that is accurate to the level of $10.0 \mathrm{MHz} \pm 0.1 \mathrm{~Hz}$. A second scaler counts the number of $10 \mathrm{MHz}$ pulses from the same $10 \mathrm{MHz}$ oscillator remaining after vetoed by the Ge dead time. The third scaler counts the number of gate pulses (GAI) that have been registered. The scalers count from zero until a clear signal is presented, and overflow after $2^{32}$ counts (approximately $400 \mathrm{~s}$ for the $10 \mathrm{MHz}$ pulses). Also included in the data readout from the ULM is a 16-bit input register that is latched at the time of the GAI. This bit register is used to capture the cycle number (bits 0-9) for the moving tape collector, the beam on/off status (bit 10), and the trigger condition (bits 11-15). The cycle number and beam on/off bits are derived from a Jorway 41 12-bit output register controlled by a Jorway 221 CAMAC timing and Sequence Module. The triggercondition bits are derived from a LeCroy E-T latch that is enabled by the Ge Master Trigger. The inputs are: $\gamma$-singles, $\gamma-\gamma$ coincidence, SCEPTAR Master Trigger, DANTE Master Trigger, and PACES Master Trigger signals. The unit is also gated by the FERA clear signal, when the Ge input lines are zero due to the Ge dead-time vetoing, so as to return the trigger-mask lines to the low state. In this way, in the event of a mistiming, the trigger condition will not register a false previous Getrigger condition but would return zero. A "good" $\gamma$-ray singles event thus will consist of an ADC header followed by a data word, the Ge-timing TDC header followed by 2 data words, a pileup-TDC header with its two data words (only if the event has a pileup flag), the BGO-timing TDC header followed by its $2 n$ data words (if there are any BGO hits), and finally the ULM header followed by its data words consisting of the bit register and the 6 data words from the 3 32-bit scalers.

\section{E.2 The SCEPTAR Data Stream}

The 20 signals from each of the SCEPTAR phototubes are fed into Phillips Scientific 776 preamplifiers that provide an amplification of $\times 10$ of the pulse voltage. One of the outputs of the preamplifier is cable-delayed by $\approx 720 \mathrm{~ns}$ and input into a LeCroy 4300 FERA for charge-to-digital conversion. The other output of the preamplifier is used for timing purposes, and fed into an Ortec 935 quad CFD with a 2 ns delay cable (the internal offset-delay jumper is positioned to provide an effective $-1.0 \mathrm{~ns}$ delay compensation, thus the equivalent delay is $1.0 \mathrm{~ns}$ ). The CFD outputs are input to a SIS3801 VME multi-channel scaler, and into CAEN 894 discriminators, the ECL outputs of which are sent to LeCroy 3377 TDC's, and the OR output from the two adjacent 894 discriminators are fanned-in to form the $\beta$ singles input to the trigger logic. A 2367 ULM is also incorporated into the SCEPTAR data stream and is operated in exactly the same mode as in the Ge data stream. A CMC203 FERA driver controls all modules, and data are 
LDRD Final Report 02-LW-026

transferred into a SIS3700 VME FIFO memory unit. The SCEPTAR data stream thus consists of a 4300-FERA header followed by $n$ data words, the SCEPTAR timing TDC header and its $2 n$ data words, and the SCEPTAR ULM header and its 7 data words.

\section{E.3 DANTE Data Stream}

The signals from the $10 \mathrm{BaF}_{2}$ detector phototubes comprise anode and dynode signals. The anode signals are used to derive timing information, are fed directly into Ortec 935 quad-CFD modules setup in the same manner as for the SCEPTAR detectors. The outputs of the CFD's are directly attached to the start inputs of Ortec 566 time-to-amplitude converters. The stop inputs are derived from the trigger condition; this can be either based on $\gamma-\gamma$ coincidences, for $\mathrm{BaF}_{2}-\mathrm{BaF}_{2}$ timing, or $\beta-\gamma$ coincidences for plastic- $\mathrm{BaF}_{2}$ timing. The outputs from the TAC's are converted in AD413a 13-bit quad-input ADC's. The $\mathrm{BaF}_{2}$ timing signals are also fed into a LeCroy 3377 TDC. The dynode signals are pre-amplified and shaped by an Ortec 113 preamplifier, followed by an Ortec 885 dual-spectroscopy amplifier, and finally converted in AD413a ADC. The DANTE data stream thus consists of the energy ADC headers and $n$ data words, the TAC ADC headers and $n$ data words, the TDC header followed by $2 n$ data words, and the ULM header followed by 7 data words.

\section{E.4 PACES Data Stream}

The signals from the $\mathrm{Si}$ detectors of PACES are fed into both an Ortec 572 spectroscopy amplifiers as well as Ortec 454 TFA's. Ortec AD413a ADC's digitize the unipolar outputs of the 572 amplifiers. Ortec $583 \mathrm{~b}$ CFD's are used to derive the time pulse from the TFA signals. The CAEN 894 discriminator provides the OR of the 5 PACES CFD signals, which is used for both the Sisingles trigger input and also as a start of a LeCroy 222 Gate and Delay Generator that provides an $\approx 1 \mu$ s wide pulse. This $1 \mu$ s pulse gates a 4532 MALU in memory enable mode with the majority output level set at 2 , thus providing the $\mathrm{Si}-\mathrm{Si}$ coincidence input for the trigger logic. The data stream is very similar to the other data streams, containing the ADC header and data, TDC header followed by its data, and the ULM header and its 7 data words.

\section{E.5 Trigger Logic}

The trigger logic is designed to provide the utmost in flexibility, while requiring the minimum in user intervention. All signals are input to a series of LeCroy 2365 Octal Logic units that can be programmed to combine any of the 16 inputs in various AND/OR combinations to 8 outputs. The inputs for the first octal logic stage are: $\gamma$-singles, $\gamma-\gamma$ coincidences, scaled-down $\gamma$-singles, $\beta$ singles, scaled-down $\beta$ singles, $\mathrm{BaF}_{2}$ singles, $\mathrm{BaF}_{2}$ coincidences, $\mathrm{Si}$ singles, scaled-down $\mathrm{Si}$ singles, and Si-Si coincidences. The outputs from this unit are those signals that will ultimately 1) form part of an OR in the final Master Trigger stage and 2) form part of a coincidence circuit with other detector subsystems. All signals are vetoed with the particular detector data-stream dead time, performed by a logical AND of the inverted dead time signal. The dead time signals are selected and inverted by a second 2365 Octal logic unit. Those signals that will form part of the coincidence circuitry are then stretched to $\approx 1 \mu \mathrm{s}$, and fed into a third octal logic unit that performs the logical AND/OR of the necessary signals and OR with the selected dead time vetoed signals from the first stage. Programming of the LeCroy 2365 Octal logic units sets the various triggers. Virtually any combination can be programmed if desired. The triggers are selected by giving a "trigger number" at the beginning of the "start-run" command.

Since one of the main programmes of research using the $8 \pi$ spectrometer and its associated auxiliary detectors is in the area of super-allowed Fermi $\beta$ decay for CKM matrix unitarity tests, a prime focus on the data acquisition system was in the ability to conduct redundant diagnosis of its performance, collecting as much information as possible on an event-by-event basis. During 
decoding of the event-stream, checks on the commonality of signals are performed. For example, the virtual station number (VSN) is encoded in the header of each ADC, there being a one-to-one relationship between the VSN and the Ge detector number. Likewise, the encoding of the channel subaddress in the fast-timing TDC allows a check that they originate from the same detector. Further checks include the matching of the event counter available from the ULM scaler data with an event counter calculated from the number of events decoded in the data stream; any mismatch in this number indicates a "dropped" event or corruption in the data itself. Thus, every trigger can be accounted for. The ULM scaler data also allows for the construction of the dead time on an event-byevent basis by examining the difference between the change in the number of $10 \mathrm{MHz}$ pulses and the change in the dead-time vetoed $10 \mathrm{MHz}$ pulses from one event to the previous event.

Also written to disk are data from the scaler modules (LeCroy 16-channel scalers) that are readout either at the end of a cycle, run, or every 10 seconds. These scalers record the number of unsuppressed Ge events, the number of suppressed Ge events, and the BGO events on a detector-by-detector basis, and various other diagnostics including the total number of events for the various arrays, the number of triggers, etc. During playback of the data, the scaler values can be checked for any anomaly, such as a spike in the hit rate that may indicate that a particular detector is firing in noise.

\section{EXPERIMENTS PERFORMED UNDER THE LDRD}

Measurements of super-allowed decays must be performed with a precision to better than $0.1 \%$ on the $f t$ value, implying that the measurements of half lives, branching ratios, and mass must be to a precision to better than $0.1 \%$. As $\gamma$-ray measurements using an array of Ge detectors have never been performed to this level before, new analysis techniques have needed to be developed. ${ }^{26} \mathrm{Na}$ beams have been used as a "standard" since its half life has been recently measured to high precision [19], and its $\gamma$-ray spectrum is dominated by the 1809 $\mathrm{keV} \quad 2^{+} \rightarrow 0^{+}$transition in ${ }^{26} \mathrm{Mg}$. One particularly difficult correction that must be applied to the data is pileup correction, but at low count rates, pileup becomes an insignificant correction. While not the case for the ${ }^{26} \mathrm{Na}$ test beam, at $10^{6} \mathrm{ion} / \mathrm{s}$, the intensity of a ${ }^{18} \mathrm{Ne}$ beam was only $10^{4} \mathrm{~s}^{-1}$. In this case, the half-life precision is limited by statistics and the dead-time correction. Figure 7 shows the results [18] for ${ }^{18} \mathrm{Ne}$ obtained by gating on the $1042-\mathrm{keV} 0^{+} \rightarrow 1^{+}$transition in ${ }^{18} \mathrm{~F}$.

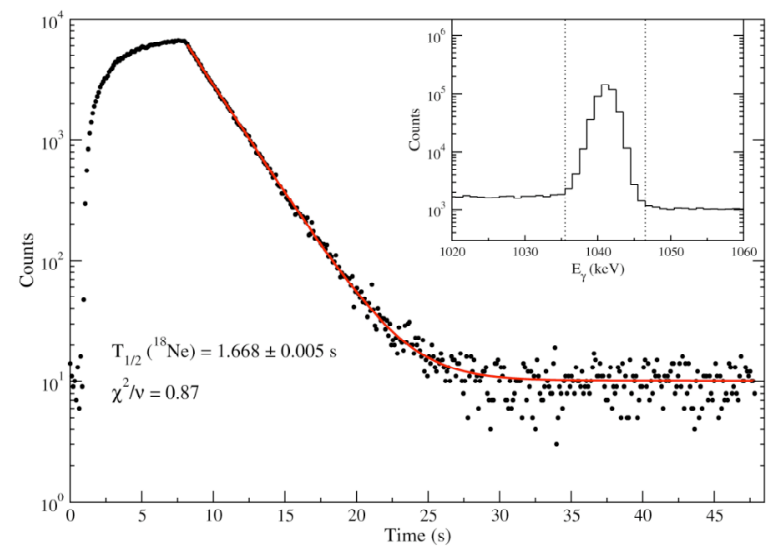

Figure 7: Half life of ${ }^{18} \mathrm{Ne}$ determined with the $8 \pi$ spectrometer. The inset shows the gate taken on the $\gamma$ ray data with the resulting time spectrum in the main portion of the figure. The curve shows the $2 \mathrm{~s}$ of background, $7 \mathrm{~s}$ of the buildup of the ${ }^{18} \mathrm{Ne}$ activity while the beam is deposited onto the tape, and then decay after $9 \mathrm{~s}$.

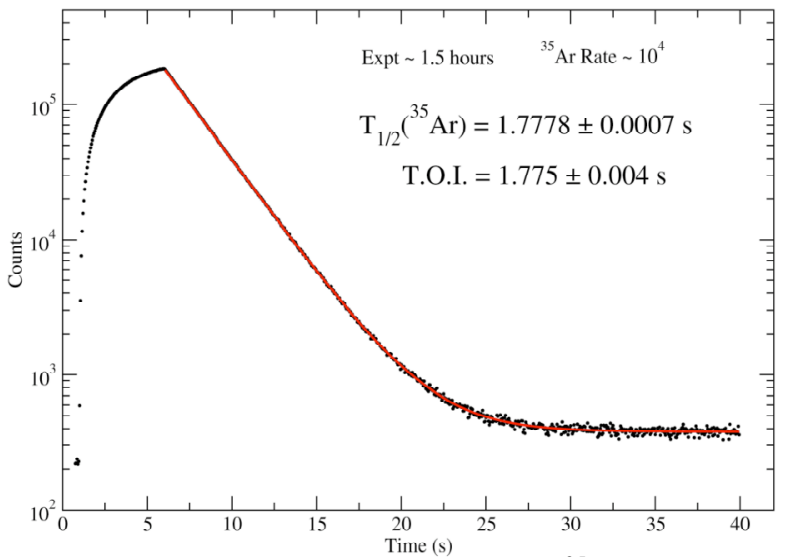

Figure 8: Very preliminary lifetime of ${ }^{35} \mathrm{Ar}$ determined with SCEPTAR. The quoted result for the uncertainty is statistical only. No corrections (dead time, etc.) have been applied. The results are already in excellent agreement with the Table of Isotopes.

A further method of measuring half lives is with the SCEPTAR array. Taking advantage of its solid angle coverage and its fast response, it 


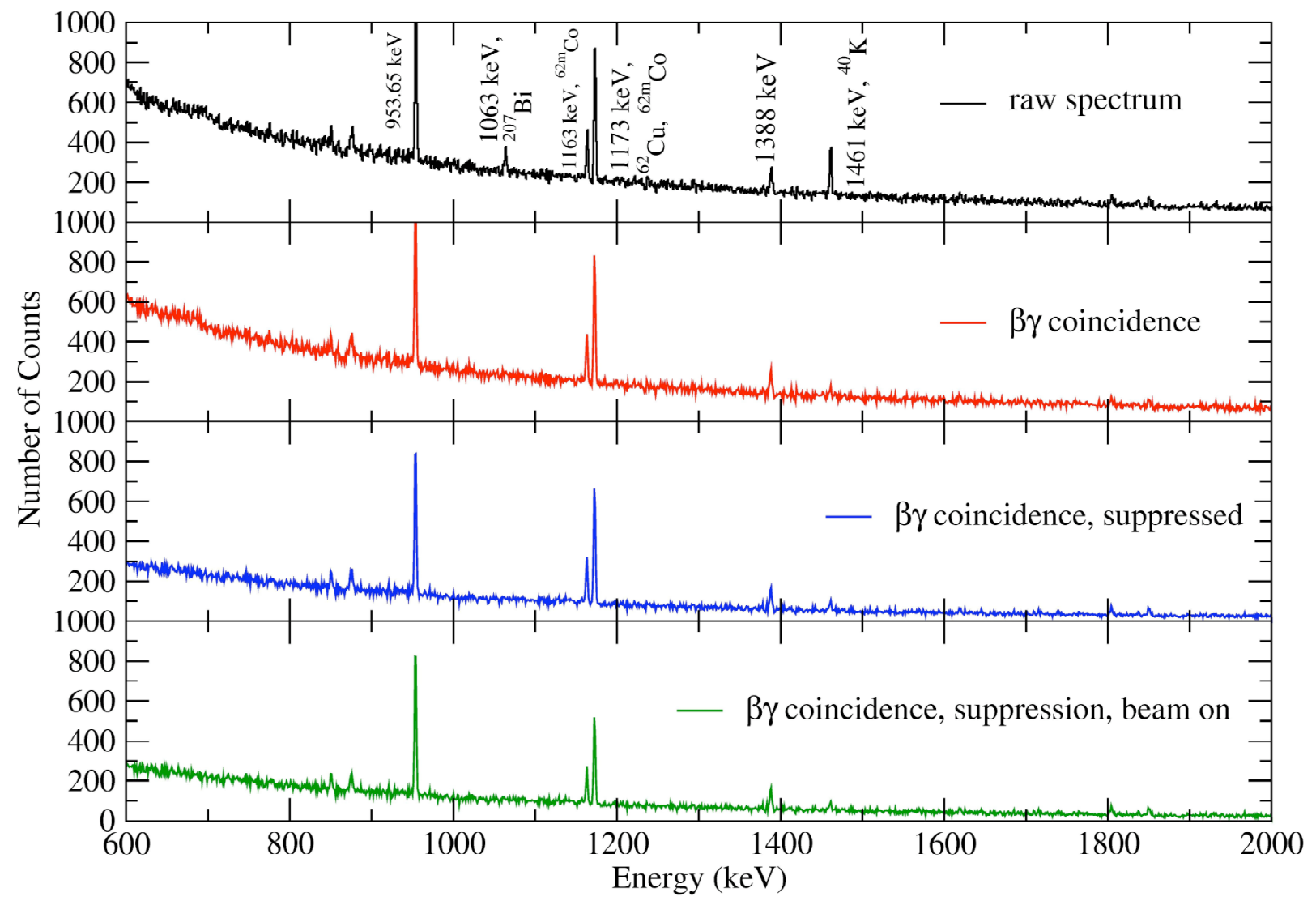

Figure 9: Portions of the $\gamma$-ray spectrum obtained with a beam of $2000 \mathrm{ion} / \mathrm{s}$ of ${ }^{62} \mathrm{Ga}$. The different panels display the improvement in the quality of the data with various conditions placed on it. The panels from top to bottom display the raw spectrum, the requirement of $\beta-\gamma$ coincidences, the application of Compton suppression, and finally that the decays occur when the $\mathrm{Ga}$ beam is being delivered to the $8 \pi$ spectrometer.

complements lifetime measurements using a $4 \pi$ gas $\beta$ counter [14]. The ability to extract half-lives with the required precision is still being investigated. Figure 8 shows very preliminary data from a recent experiment [22] examining ${ }^{35} \mathrm{Ar}$.

Branching ratio data has been obtained for ${ }^{62} \mathrm{Ga}$ decays, with a measurement using the $8 \pi$ spectrometer and a beam of $\approx 2000$ ions/s of ${ }^{62} \mathrm{Ga}$. Figure 9 displays a portion of the $\gamma$-ray spectrum collected in $\gamma$-ray singles mode with various conditions placed on the data. Using this data, a ${ }^{62} \mathrm{Zn}$ level scheme, shown in Fig. 10, was constructed. Previously, the only level observed was the first $2^{+}$level. New GamowTeller decays were observed to highly-excited states; these will constrain shell-model calculations needed for the $\delta_{C}$ correction. Analysis proceeds on this data to extract the branching ratio to high precision.

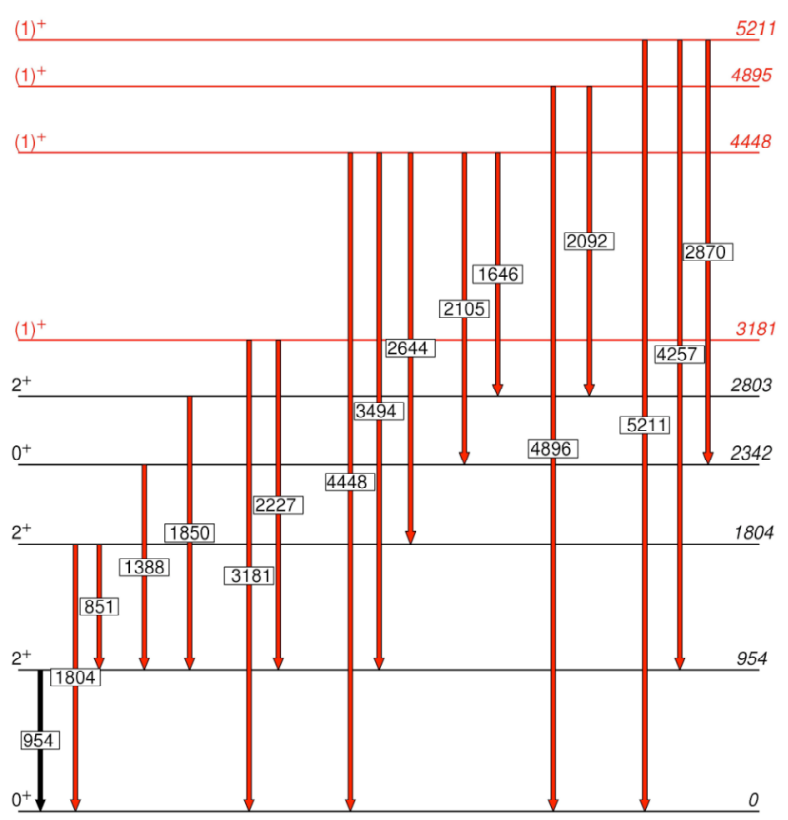

Figure 10: Level scheme of ${ }^{62} \mathrm{Zn}$ obtained from the ${ }^{62} \mathrm{Ga}$ super-allowed $\beta$ decay. Only the $954-\mathrm{keV} 2^{+}$level was observed in previous $\beta$ decay studies. New GamowTeller decays were observed up to an excitation energy of $5.2 \mathrm{MeV}$. 


\section{LDRD Final Report 02-LW-026}

The progress of the experimental program was hampered by the lack of timely availability of radioactive beams from the TRIUMF facility. The development of new radioactive beams is a problem common to all radioactive beam facilities, and generally it is acknowledged that it is far more difficult than originally thought. For example, ${ }^{34} \mathrm{Ar}$, the measurement that was to be performed in the first year of this LDRD, was believed to be a readily available beam. Being a noble gas, it diffuses easily out of the production target. However, construction of a working ECR source that was capable of handling the gas loads in such an environment proved to be very difficult. It is currently thought that a new FEBIAD source to be tested at TRIUMF in 2006 will be sufficiently efficient such that a beam of $10^{5}{ }^{34} \mathrm{Ar} / \mathrm{s}$ will be achievable.

While the ${ }^{34} \mathrm{Ar}$ measurements should occur in 2006, the LLNL led experiments on ${ }^{66} \mathrm{As}$ and ${ }^{70} \mathrm{Br}$ may not occur until suitable ion sources come on line, perhaps in 2007 and 2008. Long-term involvement of LLNL personnel will be required to lead these experiments and profit from the investment begun with this LDRD.

\section{BENEFITS TO LLNL}

The measurements at TRIUMF involve an international collaboration from institutions in both Canada and the US. The experimental program has been given the highest priority by the TRIUMF PAC, and the NSAC Long Range Plan highlighted $\beta$-decay tests of the Standard Model. The LLNL contribution has had a high impact, and is highly visible. Tests of the Standard Model are very high profile, and if unitarity is shown to be disobeyed it would attract much attention to all of the participants. The involvement of LLNL in the $\gamma$-ray spectroscopy program at the "ground-level" has tremendous benefit for $\mathrm{N}$-Division personnel. New experimental programs have been developed, including the measurement of very short-lived nuclear lifetimes with electronic timing techniques, and now involvement in accelerated radioactive beam programs with the TIGRESS collaboration at TRIUMF/ISAC-II.

\section{CONCLUSIONS}

The $8 \pi$ spectrometer and its auxiliary detectors, SCEPTAR, DANTE, and PACES, together with the Moving Tape Collector and a powerful and versatile data acquisition system, is the world's most powerful device devoted to radioactive decay studies, and is a unique tool for nuclear physics studies. Many studies using the $8 p$ spectrometer involving LLNL personnel to date have included: 1 ) the $\beta$ decay of ${ }^{11} \mathrm{Li}$, to study the possibility of neutron-halo "survival" during the $\beta$ decay process [23], 2) ${ }^{18} \mathrm{Ne}$ [18], 3) ${ }^{35} \mathrm{Ar}[22]$, and 4) ${ }^{62} \mathrm{Ga}$ [19], as part of the super-allowed Fermi $\beta$-decay programme, 5) ${ }^{26} \mathrm{Na}$ [19], (our workhorse test beam), 6) ${ }^{32} \mathrm{Na}$ to investigate the structure of ${ }^{32} \mathrm{Mg}$ and the island of inversion, 7) ${ }^{156} \mathrm{Ho}$ and 8) ${ }^{158} \mathrm{Tm}$ decay as part of the programme to study shapephase transitions in the $N=90$ isotones, and isomeric states in 9) $\left.{ }^{174} \mathrm{Tm}[20,21], 10\right){ }^{178} \mathrm{Hf}$ [24], and 11) ${ }^{178,179} \mathrm{Lu}$ [25]. Accepted LLNLled proposals include a study of the superallowed Fermi $\beta$ decays of ${ }^{66} \mathrm{As}$ and ${ }^{70} \mathrm{Br}$, and the nuclear structure of neutron-rich $\mathrm{Cd}$ and $\mathrm{Pd}$ isotopes in connection with $r$-process nucleosynthesis in the mass 120 region. This list will continue to grow as ISAC develops more production targets and ion sources to provide a richer variety of radioactive beams.

\section{ACKNOWLEDGEMENTS}

Work performed under the auspices of the US Department of Energy by the University of California, Lawrence Livermore National Laboratory under contract no. W-7405-ENG48. 
[1] I. Towner and J.C. Hardy, Phys. Rev C 66, 035501 (2002).

[2] J.C. Hardy and I.S. Towner, Phys. Rev. C 71, 055501 (2005).

[3] H. Abele et al., Phys. Rev. Lett. 88, 211801 (2002).

[4] A. Sher et al., Phys. Rev. Lett. 91, 261802 (2003).

[5] S. Eidelman et al. Phys. Lett. B592, 1 (2004).

[6] G. Isidori, Proc. $2^{\text {nd }}$ conference on CKM Unitarity Triangle, Durham, UK (2003) WG6, eConfC0304052 hep-ph/0311044

[7] A. Alexopoulous, et al., Phys. Rev. Lett. 93, 181802 (2004).

[8] N. Cabibbo et al., Phys. Rev. Lett. 92, 251803 (2004).

[9] A. Lai et al., Phys. Lett B602, 41 (2004).

[10] J. C. Hardy and I. S. Towner, Phys. Rev. Lett. 94, 92502 (2005).

[11] W.E. Ormand and B.A. Brown, Nucl. Phys. A440, 274 (1985).

[12] W.E. Ormand and B.A. Brown, Phys. Rev. Lett. 62, 866 (1989).

[13] W.E. Ormand and B.A. Brown, Phys. Rev. C 52, 2455 (1995).

[14] G.C. Ball, et al., Phys. Rev. Lett. 86, 1454 (2001).

[15] A. Piechaczak et al. Phys. Rev. C 67, 051305 (2003).

[16] B. Blank et al., Phys. Rev. C 69, 015502 (2004).

[17] B. Hyland et al., J. Phys. G 31, S1885 (2005).

[18] M.B. Smith, G.F. Grinyer, et al., to be published.

[19] G.F. Grinyer, et al., Phys. Rev. C 71, 044309 (2005).

[20] R.S. Chakrawarthy, et al., The Fourth International Conference on Exotic Nuclei and Atomic Masses, ENAM'04, Pine Mountain, Georgia, Sept. 2004, Eur. Phys. J. A25 S01, 125 (2005).

[21] R.S. Chakrawarthy, et al., to be published.

[22] G.F. Grinyer, et al., to be published.

[23] F. Sarazin, et al., Phys. Rev. C 70, 031302 (2004).

[24] M.B. Smith, Phys. Rev. C 68, 031302(R) (2003).

[25] M.B. Smith et. al., Nucl. Phys. A746, 617c (2004).

\section{$\underline{\text { PUBLICATIONS ASSOCIATED WITH LDRD }}$}

[1] P.E. Garrett, et al., UCRL-PROP-151366

[2] G.F. Grinyer, C.E. Svensson, C. Andreoiu, A.N. Andreyev, R.A.E. Austin, G.C. Ball, R.S.

Chakrawarthy, P. Finlay, P.E. Garrett, G. Hackman, J.C. Hardy, B. Hyland, V. Iacob, K.A.

Koopmans, W.D. Kulp, J.R. Leslie, J.A. MacDonald, A.C. Morton, W.E. Ormand, C.J. Osborne, C.J. Pearson, A.A. Phillips, F. Sarazin, M.A. Schumaker, H.C. Scraggs, J. Schwarzenberg, M.B. Smith, J.J. Valiente-Dubon, J.C. Waddington, J.L. Wood, and E.F. Zganjar, et al., Phys. Rev. C 71, 044309 (2005).

[3] R.S. Chakrawarthy, P.W. Walker, M.B. Smith, A.N. Andreyev, S.F. Ashley, G.C. Ball, J.A. Becker, J.J. Daoud, P.E. Garrett, G. Hackman, G. Jones, Y. Litvinov, A.C. Morton, C.J. Pearson, C.E. Svensson, S.J. Williams, and E.F. Zganjar, The Fourth International Conference on Exotic Nuclei and Atomic Masses, ENAM'04, Pine Mountain, Georgia, Sept. 2004, Eur. Phys. J. A25 S01, 125 (2005). UCRL-PROC-208879

[4] F. Sarazin, J.S. Al-Khalili, G.C. Ball, G. Hackman, P.M. Walker, R.A.E. Austin, B. Eshpeter, P. Finlay, P.E. Garrett, G.F. Grinyer, K.A. Koopmans, W.D. Kulp, J.R. Leslie, D. Melconian, C.J. Osbourne, M.A. Schumaker, H.C. Scraggs, J. Schwarzenberg, M.B. Smith, C.E. Svensson, J.C. Waddington, and J.L. Wood, Phys. Rev. C 70, 031302 (2004).

[5] M.B. Smith, P.M. Walker, G.C. Ball, J.J. Carroll, P.E. Garrett, G. Hackman, R. Propri, F. Sarazin, and H.C. Scraggs, Phys. Rev. C 68, 031302(R) (2003). 
[6] M.B. Smith, P.M. Walker, G.C. Ball, J.J. Carroll, P.E. Garrett, G. Hackman, R. Propri, F. Sarazin, and H.C. Scraggs, The Sixth International Conference on Radioactive Nuclear Beams (RNB6), 22-26 Sept. 2003, Argonne, USA UCRL-TR-202881

[7] W.D. Kulp, et al., Phys. Rev. C 71, 041303 (2005), UCRL-JRNL-210144.

[8] C.E. Svensson, R.A.E. Austin, G.C. Ball, P. Finlay, P.E. Garrett, G.F. Grinyer, G.S. Hackman, C.J. Osborne, F. Sarazin, H.C. Scraggs, M.B. Smith, and J.C. Waddington, EMIS-14: Fourteenth International Conference on Electromagnetic Isotope Separators and their Applications, May 610, 2002, Victoria, Canada, Nucl. Instrum. Meth. in Phys. Res. B204 660 (2003).

[9] W.D. Kulp, J. L. Wood, J. Allmond, P.E. Garrett, D. Cline, C.Y. Wu, A.B. Hayes, H. Hua, R. Teng, 2002 Fall Meeting of the Division of Nuclear Physics of the American Physical Society, East Lansing, USA, Oct. 2002 Bull. Am. Phys. Soc. 47, no.6, 93 (2002).

[10] P.E. Garrett, G.S. Hackman, P.-A. Amaudruz, G.C. Ball, C.J. Osborne, F. Sarazin, H.C. Scraggs, M.B. Smith, C.E. Svensson, P. Finlay, G.F. Grinyer, J.C. Waddington, R.A.E. Austin, W.D. Kulp, J.L. Wood, R. Sumner, 2002 Fall Meeting of the Division of Nuclear Physics of the American Physical Society, East Lansing, USA, Oct. 2002 Bull. Am. Phys. Soc. 47, no.6, 101 (2002).

[11] J.L. Wood, W.D. Kulp, J. Allmond, P.E. Garrett, D. Cline, C.Y. Wu, A.B. Hayes, H. Hua, R. Teng, 2003 Fall Meeting of the Division of Nuclear Physics of the American Physical Society, Touscon, USA, Oct. 2003 Bull. Am. Phys. Soc. (2003).

[12] F. Sarazin, G.C. Ball, G. Hackman, J.S. Al-Khalili, P.M. Walker, B. Eshpeter, D. Melconian, C.J. Osbourne, H.C. Scraggs, M.B. Smith, R.A.E. Austin, J.C. Waddington, P.E. Garrett, G.F. Grinyer, C.E. Svensson, W.D. Kulp, J.L. Wood, J.R. Leslie, 2004 Spring Meeting of the American Physical Society, Denver, USA, May 2004 Bull. Am. Phys. Soc. (2004).

[13] P.E. Garrett, W.D. Kulp, J.L. Wood, 2004 Fall Meeting of the Division of Nuclear Physics of the American Physical Society, Chicago, USA, Oct. 2004 Bull. Am. Phys. Soc. (2004).

[14] W.D. Kulp, J.L. Wood, P.E. Garrett, 2004 Fall Meeting of the Division of Nuclear Physics of the American Physical Society, Chicago, USA, Oct. 2004 Bull. Am. Phys. Soc. (2004).

[15] C.M. Mattoon, F. Sarazin, G.C. Ball, E.S. Cunningham, G. Hackman, S.C. Ravuri, H.C. Scraggs, M.B. Smith, R.A.E. Austin, K.A. Koopmans, J.C. Waddington, B. Washbrook, P. Finlay, B. Hyland, A.A. Phillips, M.A. Schumaker, C.E. Svensson, P.E. Garrett, J.R. Leslie,

J. Schwarzenberg, P.M. Walker, E. Zganjar, 2004 Fall Meeting of the Division of Nuclear Physics of the American Physical Society, Chicago, USA, Oct. 2004 Bull. Am. Phys. Soc. (2004).

[16] G.C. Ball, T. Achtzein, D. Albers, J.A. Al Khalili, C. Andreoiu, A. Andreyev, S.F. Ashley, R.A.E. Austin, J.A. Becker, P. Bricault, S. Chan, R.S. Chakrawarthy, R. Churchman, H. Coombes, E.S. Cunningham, J. Daoud, M. Dombsky, T.E. Drake, B. Eshpeter, P. Finlay, P.E. Garrett, C. Geppert, G.F. Grinyer, G. Hackman, V. Hanemaayer, B.H. Hyland, G.A. Jones, K.A. Koopmans, W.D. Kulp, J. Lassen, J.P. Lavoie, J.R. Leslie, Y. Litvoniv, J.A. MacDonald, C. Mattoon, D. Melconian, A.C. Morton, C.J. Osbourne, C.J. Pearson, M. Pearson, A.A. Phillips, P.H. Regan, J.J. Ressler, F. Sarazin, M.A. Schumaker, J. Scharzenberg, H.C. Scraggs, M.B. Smith, C.E. Svensson, J.J. Valiente-Dubon, J.C. Waddington, P.M. Walker, K. Wendt, S.J. Williams, J.L. Wood, and E.F. Zganjar, International conference on Nuclear Structure, Astrophysics and Reactions, NUSTAR'05, Surrey, UK, Jan. 2005, J. Phys. G 31 S1491 (2005).

[17] S.J. Williams, P.M. Jenneson, P.E. Garrett, P.H. Regan, C. Andreoiu, G.C. Ball, R.S. Chakrawarthy, J.J. Daoud, G.F. Grinyer, G. Hackman, B. Hyland, N.A. Mlwilo, A.C. Morton, C.J. Pearson, M.A. Schmaker, M.B. Smith, C.E. Svensson, and J.J. Valiente-Dubon, International conference on NUclear STructure, Astrophysics and Reactions, NUSTAR'05, Surrey, UK, Jan. 2005, J. Phys. G 31, S1979 (2005).

[18] P.E. Garrett, W.D, Kulp, J.L. Wood, D. Bandyopadhyay, S. Christen, S. Choudry, A. Dewald, A. Fitzler, C. Fransen, K. Jessen, J. Jolie, A. Kloezer, P. Kudejova, A. Kumar, S.R. Lescher, A. Linnemann, A. Lisetskiy, D. Martin, M. Masur, M.T. McEllistrem, O. Moller, M. Mynk, J.N. Orce, P. Pejovic,T. Pissulla, J.-M. Regis, A. Schiller, D. Tonev, and S.W. Yates, 
LDRD Final Report 02-LW-026

International conference on Nuclear Structure, Astrophysics and Reactions, NUSTAR'05, Surrey, UK, Jan. 2005, J. Phys. G 31, S1855 (2005). (UCRL-JRNL-213397) 